\title{
ANTE UNA DISYUNTIVA: ECONOMÍA DE LA ACUMULACIÓN Y EL DERROCHE O ECONOMÍA DE LO JUSTO Y AUSTERO
}

Jorge Flores Silva. Universidad Nacional Autónoma de Honduras, Facultad de Ciencias Económicas, Instituto de Investigaciones Económicas y Sociales (UNAH-FCE-IIES), Ciudad Universitaria, Edifício C2, Primer piso, Tel/Fax: 504-2239 1849

E-mail: jflores@iies-unah.org

\section{RESUMEN}

Los que controlan el capital como resultado de una economía de acumulación, son los que tienen la posibilidad de hacer las inversiones, lo hacen en aquello que les da mayor rentabilidad en función del interés privado y no del interés común.

El problema del modelo de acumulación en los actuales momentos se expresa con la crisis de producción de alimentos, el desempleo y la pobreza que vive la población, fenómeno que tiene varias causas, pero sus raíces principales son de carácter estructural. Se está ante una estructura económica de concentración y una superestructura política de injusticia.

A diferencia de una economía de la acumulación en la economía de lo justo, se pueden introducir criterios de solidaridad y el bien común en donde el fin último ha de ser el sujeto de la actividad económica -el ser humano- y el dinero y la tecnología solo han de ser un medio para lograr la realización del individuo como centro y sujeto de la actividad productiva.

Palabras Claves: Acumulación, economía de la acumulación, derroche. 


\section{FACED WITH A CHOICE: ECONOMICS OF WASTE ACCUMULATION AND THE ECONOMY OR WHAT IS JUST AND AUSTERE}

Jorge Flores Silva. Universidad Nacional Autónoma de Honduras, Facultad de Ciencias Económicas, Instituto de Investigaciones Económicas y Sociales (IIES-UNAH), Ciudad Universitaria, Edifício C2, Primer piso, Tel/Fax: 504-2239 1849

E-mail: jflores@iies-unah.org

\section{ABSTRACT}

The nerves that control the capital as a result of an economy of accumulation, are the ones who have the possibility of making the investment, they do so in something that gives them more profitability in function of the private interest and not the common interest.

The problem of model of accumulation in the current moment is expressed with the crisis in food production, unemployment and poverty that people live, a phenomenon that has several causes, but their main roots are structural in nature.

Unlike an economy based on the accumulation in the economy of what is right, you can enter criteria of solidarity and the common good in where the ultimate goal has to be the subject of economic activity - the human being - and the money and the technology only have to be a means for the realization of the individual as the center and subject of productive activity.

Keywords: Accumulation, economy of accumulation, waste. 


\section{INTRODUCCIÓN}

La economía de acumulación basada en la máxima ganancia enfatiza la apertura de mercados como forma de insertarse en el intercambio mundial. Las condiciones para ese intercambio ventajoso supuestamente descansan en el irrestricto respeto a los principios del mercado.

En el marco de las relaciones de intercambio, destaca la suscripción y puesta en vigencia tratados comerciales, cuya esencia radica en el mejoramiento de las condiciones competitivas para mantener un intercambio comercial recíprocamente ventajoso.

La política que se impulsa responde a un modelo, cuyas premisas fundamentales apuntan más a resolver problemas coyunturales, soslayando los cambios estructurales necesarios para el desarrollo económico que tenga como objetivo la reproducción de la naturaleza en función de la vida. Un desarrollo económico y social que esté centrado en el desarrollo de la persona.

E\&A

Los cambios sobre la base de un modelo de desarrollo, es clave para posibilitar el incremento de la producción y la productividad, en torno a la cual se ensanche la base material de la sociedad y genere una oferta interna que estimule la actividad productiva y vuelva más dinámico el comercio exterior, aprovechando las ventajas competitivas que puedan ser resultado de un proceso educativo que humanice y socialice, para servir de instrumento de transformación y de modificación de patrones de conducta y de consumo para lograr el progreso social.

A pesar de contar con recursos naturales y humanos, la falta de una clara visión sobre la problemática del desarrollo nos ha llevado a una profundización de las ya graves condiciones de rezago de la economía, con los consecuentes efectos en los niveles de vida de la población. 


\section{EL PROBLEMA DEL DESARROLLO Y EL MODO DE FUNCIONAR DE LA ECONOMÍA}

El problema del desarrollo implica un proceso global cuyo tratamiento debe conducir a superar los desequilibrios de la economía, que posibilite un proceso de desarrollo social que impulse un modelo de desarrollo con justicia y equidad, sobre la base de una estrategia que garantice la sostenibilidad del proceso.

Resulta urgente y necesario la reflexión y el debate, así como la postulación de ideas para la ejecución de medidas encaminadas a transformar la economía, transformando las formas de trabajo, las formas de explotar los recursos y los hábitos de consumo.

El uso de los recursos y la forma de apropiación de éstos, tiene su efecto sobre la economía e incide sobre el bienestar de la población.

El modo de funcionar de la economía, no solo ha gestado un régimen deficiente, sino que ha dado lugar a un sistema de producción orientado a la especulación y el lucro con mercados externos que no dan garantía de estabilidad.

Los que controlan el capital como resultado de una economía de acumulación, son los que tienen la posibilidad de hacer las inversiones, lo hacen en aquello que les da mayor rentabilidad en función del interés privado y no del interés común.

El problema del modelo de acumulación en los actuales momentos se expresa con la crisis de producción de alimentos, el desempleo y la pobreza que vive la población, fenómeno que tiene varias causas, pero sus raíces principales son de carácter estructural. Se está ante una estructura económica de concentración y una superestructura política de injusticia.

Según el destacado economista Roberto Verrier Castro vicepresidente primero de la Asociación de Economistas de América Latina y el Caribe, se sintetizan algunos datos que él da a conocer en su 
interesante trabajo "América Latina: el descalabro del modelo neoliberal, y la crisis en el corazón de la economía norteamericana" los cuales indican que: el ingreso de las 500 personas más ricas del mundo, es superior al ingreso de los 416 millones de personas más pobres. Los 33 hombres de negocios más ricos de Latinoamérica aglutinan una fortuna cercana a los 114 mil millones de dólares, igual al ingreso que reciben 160 millones de pobres que subsisten con menos de dos dólares diarios. El mismo Profesor Verrier haciendo referencia a publicaciones de revistas y diarios expresa que los tres multimillonarios que encabezan la lista de los ricos del planeta poseen más activos que el PIB de los 48 países más pobres del mundo. Si se emplease todo el dinero que se emplea en armamentos, se podría alimentar a toda la población que hoy no come. El gasto de la lucha contra el SIDA que cobra millones de vidas al año, equivale a tres días del gasto militar del mundo".

La política educativa también se subordina a la política económica que afianza el subdesarrollo, las formas de trabajo y hábitos de consumo que promueve, y el mensaje e imagen única que da a conocer imposibilita generar una conciencia crítica y organizativa con espíritu solidario, para una conciente participación en las grandes decisiones nacionales.

El modelo de acumulación que busca ya no la ganancia sino la máxima ganancia, en contraposición al modelo de economía justa y austera, "genera riqueza" destruyendo las fuentes que producen riqueza. Esto implica un costo ecológico, económico y social, por la ruptura del equilibrio natural que no garantiza un desarrollo sostenible, por cuanto se hace en forma desordenada, sin un efectivo manejo que asegure su conservación y buen aprovechamiento.

Las nuevas formas de organización de la Economía en el marco de un proceso de globalización los países se integran a través del comercio, el avance tecnológico y los flujos financieros. Urge entonces aprovechar las ventajas competitivas que tienen los países, sobre todo su riqueza natura y el potencial productivo del recurso humano, el cual aprovechado de manera racional y humana puede permitir gestar un proceso de desarrollo que involucre a todos los actores del progreso social.

la capacidad de respuesta que puedan dar los actores involucrados en un proceso de transformación que se inscriba en el marco de una economía justa y austera dependerá de que se consideren aspectos 
fundamentales relacionados con: la explotación de los recursos naturales, aprovechando sus propios limites para el progreso y el desarrollo económico; significado del mercado y los patrones de consumo en el ámbito socioeconómico y sus implicaciones en la economía en su conjunto; papel de la tecnología sobre la producción y la productividad; papel del Estado y la política económica en la orientación de la actividad productiva; la educación como practica social que genera procesos humanos para el desarrollo y la expansión económica.

El proceso de globalización no es un fenómeno nuevo, es resultado del modo de funcionar del capitalismo, siendo que el capital y las relaciones que se derivan de este modo de producción siempre han tenido vocación internacional. Es a principios de la década de los 90s que se empieza hablar de manera generalizada del fenómeno globalización y tomó mayor postura con la caída del socialismo de Europa del Este. Se habla dentro de todo este proceso del neoliberalismo, del mercado total y del totalitarismo del mercado.

El proceso de globalidad no es reciente, es producto de procesos históricos anteriores y que ahora aparecen como una nueva etapa en el desarrollo del capitalismo, no se da en los años noventa, pero sí el termino surge en los años noventa, la globalización aunque la vemos como una estrategia impuesta al mundo por los organismos multilaterales, pero en si no es impuesta por estos organismos, sino por los grupos de poder económico y político que tienen y ejercen influencia en estos organismos. Antes hubo, como dice F. Hinkelammer, toda una lucha de palabras, para imposibilitar los cambios, porque siempre el lenguaje juega un papel importante para impulsar los cambios o para frenar los mismos, entonces la lucha se ha librado para evitar un pensamiento alternativo, se habla entonces de teoría global a lo que sobreviene la critica y se alzan las voces para una teoría especifica como la teoría de la dependencia de las décadas de los sesenta y setenta. Posteriormente se habla de reforma, primero eran cambios frente al mercado capitalista, de este pensamiento de reforma se le da vida al sistema de planificación económica como forma de influir deliberadamente en el funcionamiento del sistema económico. En si la globalización como dice Maryse Brisson (1999) “... es una exigencia de la necesidad de siempre hacer más ganancia; es una manera de responder a los problemas que fueron surgiendo. Es una respuesta para desmantelar una forma de resistencia. Es una manera de doblegar tanto a la fuerza de trabajo como a la supuesta soberanía de las naciones" p. 68 y 69. 
Si el proceso económico tiene como objetivo la máxima ganancia por la vía de la especulación y el lucro, los criterios de la política económica en un proceso económico que busca la utilidad sin atender al bien común van a circunscribirse a: producir según las utilidades de las personas, empresa que produce según la demanda lo que él quiera - y esto exige producir u ofrecer lo que él quiera. De ahí que el criterio básico es la ganancia sobre la base del mercado libre. Parafraseando a W. Hugues.

"Si en el proceso económico el objetivo fundamental es el bienestar de las personas, es la vida humana, entonces la máxima ganancia no interesa, no es necesaria, es más no podría lograrse, ya que lo que interesa es el bien común, el ser humano, su dignidad, su inclusión en las grandes decisiones. El crecimiento económico bajo esta perspectiva pasa a ser un medio en función del bien común y el interés social, generando una estructura de distribución con equidad porque sus aspectos cualitativos se aprovechan en beneficio y función de la mayoría de las personas". En todo este proceso el ser humano es el sujeto que da vida y dinamiza la actividad económica, es imposible hablar de desarrollo sin incluir a la persona que es el sujeto de toda acción humana e institucional. con los demás, que lo llevan a buscar y descubrir las virtudes de los demás, a dimensionar el principio y valor de la solidaridad, dejando huella positiva que trasciende en el tiempo, poniendo su inteligencia, conocimiento y capacidad al servicio del bien común. También mantiene relaciones con la naturaleza las que dándose con responsabilidad, guardan el equilibrio para su propia existencia. El ser humano y su actividad transformadora siempre ha tenido gran influencia en los procesos de cambio que sufre la tierra y lo que habita en ella, causando gran impacto sobre el medio ambiente no solo a nivel local sino también a escala y dimensión global. La presión sobre los recursos naturales y el avance técnico-científico no siempre ha estado regulada para que su aplicación sea rehabilitador y conservador de los recursos provenientes de la casa de todos -la Tierra-. De ahí la necesidad de una normativa basada en una ética que postule una ciencia y una técnica al servicio del ser humano y al cuidado de la naturaleza. Es responsabilidad de todos conocer e informarse sobre los cambios del sistema terrestre, el impacto del medio ambiente y su efecto sobre la vida de las personas y su entorno. 
El examen critico del problema de la tierra lleva a concluir que el desequilibrio ecológico es resultado de la acción humana, producto de una conciencia antiecológica que privilegia el tener por sobre el ser, promoviendo un estilo de vida consumista, que lleva al ser humano a producir y consumir, en donde los valores supremos son las leyes del mercado, la eficiencia y la competitividad en función del utilitarismo para el aparato productivo y las exigencias del mercado, en detrimento de la naturaleza y los recursos que ella provee.

Estamos frente a un modelo de acumulación y consumo basado en la especulación que instrumentaliza al ser humano y deteriora la naturaleza. Este fenómeno económico basado en el lucro y la máxima ganancia, hace que los bienes de consumo que se producen tengan una vida corta, que luego se echan al cesto de la basura como resultado del derroche. De esa manera se estaría destruyendo más rápidamente la naturaleza, porque es de la naturaleza de donde se obtiene la materia prima para producirlos.

La estrategia económica que se impulsa sobre la base de un modelo de desarrollo para la acumulación y el consumismo no solo destruye la naturaleza, sino al ser humano mismo, en donde lo que priva es la eficiencia para competir, se privilegia una política de crecimiento en la que hay que producir para consumir, el ser humano se cosifica y el curriculum educativo que es parte de ese modelo, prepara al individuo para hacer cosas.

Así como la política educativa se subordina a la política económica, también el ser humano se somete al capital. El capital deja de ser un medio para dignificar al hombre, convirtiéndose más bien en un fin, en función de generar la máxima ganancia por la vía del lucro y la especulación. Priva el crecimiento que se concibe como un fin en si mismo y se subestima la política que tiene como centro y fin la vida del ser humano.

El modo de funcionar de la economía, en la que el capital lo subordina todo, priva por sobre el mismo ser humano el criterio de la máxima ganancia, haciendo depender del mercado a la población. Penetra además diferentes áreas de producción y de consumo de mercancías que bajo distintas formas hacen más necesario los medios de pago y la sustitución del trabajo artesanal independiente, por el trabajo asalariado y el subempleo visible e invisible. 


\section{HACIA UNA ECONOMÍA DE LO JUSTO Y AUSTERO O DEL BIEN COMÚN}

A diferencia de una economía de la acumulación en la economía de lo justo, se pueden introducir criterios de solidaridad y el bien común en donde el fin último ha de ser el sujeto de la actividad económica -el ser humano- y el dinero y la tecnología solo han de ser un medio para lograr la realización del individuo como centro y sujeto de la actividad productiva.

La participación de la persona como sujeto social, es una de las características del trabajo en una economía de lo justo y austero, mediante la cual se busca el bien común a través del trabajo organizado y solidario. En tal sentido, es más importante el ser humano que el propio dinero que puedan aportar para la consecución de sus fines económicos y sociales.

El Estado que no debe renunciar a su deber de velar por el bien común, la sociedad civil a través de las organizaciones no gubernamentales como parte también del Estado y la cooperación internacional deben estar siempre impulsando el trabajo solidario, aspirando siempre a la valoración y desarrollo de la persona, abogando e impulsando la justicia, el respeto a la dignidad humana y la práctica del trabajo planificado.

Esta concepción de la economía de lo justo y las características de las unidades económicas que de ella se generen, implica que lo fundamental en esta forma de producción y organización del trabajo es el carácter solidario de sus integrantes y que sus actividades económicas y sociales deben realizarse para provecho de sus miembros y de la comunidad porque generan riqueza que deben ser utilizadas para mejorar las condiciones de vida de la sociedad en general. Lo fundamental en todo esto es crear la cultura de la solidaridad, como dice el profesor W. Dierkxsens o somos solidarios con el otro o nos morimos todos. Además deben dársele a la gente las herramientas necesarias para que pueda desarrollarse y logre de esa forma su autorrealización a través del trabajo productivo que lo dignifica.

El trabajo solidario en el marco de una economía de lo justo y austera ha de facilitar la gestión empresarial y capacidad creadora para la producción de bienes y servicios que fortalezcan el aparato productivo y la racionalidad social. 
En las condiciones actuales se está ante la disyuntiva o se favorece una estructura industrial-comercial para los sectores de altos ingresos que generalmente demanda de bienes importados cuyos efectos se ven reflejados en la balanza comercial o se da la primacía a las necesidades globales de la sociedad que evidentemente no tiene capacidad de compra y está fuera de las relaciones de mercado.

En una economía basada en una racionalidad formal cuyo objetivo es la ganancia, y no solo la ganancia sino la máxima ganancia, no interesa el hecho de que la mayoría de la población no tenga capacidad de ahorro, ni acceso al mercado porque basta a la lógica del funcionamiento de la economía mercantil que un sector tenga poder de compra y sus necesidades se expresen en termino monetarios para que se cumplan sus objetivos, independientemente del tipo de productos que demanden.

Las distorsiones que esto genera en la estructura comercial y de consumo que se implanta tienen su expresión concreta en el tipo de bienes que demanden los que tienen acceso al ingreso. Los grupos de altos ingresos tienen costumbres y patrones que no responden a una economía atrasada y por lo mismo los bienes y servicios que consumen son propios de países industrializados muy diferentes a los productos que pueda demandar la población mayoritariamente pobre.

Por las condiciones propias del modelo de desarrollo, los distintos factores resultan estar siempre ligados. El problema del empleo, la producción, la productividad y la racionalidad en el uso y explotación de los recursos está estrechamente ligado al grado de desarrollo tecnológico que pueda experimentar la sociedad. No se critica el avance técnico-científico que observa el mundo actual, la crítica que se hace es que no se emplea guardando la racionalidad para evitar el atraso. El uso o no de tecnología racionalizadora dependerá de la visión que se tenga de la problemática del desarrollo y de los objetivos del proceso económico. Si el propósito solo es el incremento de la productividad, como resultado de una relación de eficiencia en el uso de los recursos, quizá se obvie su importancia socioeconómica como resultado de la distribución de los beneficios del progreso técnico a favor de todos los sectores sociales, incluidos los más pobres. 
Si se quiere favorecer a los pobres, si ha de crearse empleos y apegarse a los elementos sociales y económicos que debe postular la técnica, para que esté al servicio de los seres humanos, ha de considerarse nuevas opciones en la elección del tipo de tecnología a emplear.

Las preguntas que surgen en torno a esto son: ¿qué tipo de técnicas e innovación tecnológica interesa para favorecer el bien común? Si el objetivo es la maximización ¿quiénes saldrían favorecidos, la sociedad toda o reducidos grupos con un interés particular e individual? la realidad no deja duda que la aplicación de nuevas técnicas, sin una distribución, solo reconcentra los beneficios del progreso y empeora las condiciones de vida de la gente, principalmente de los excluidos. En un proceso de desarrollo, dentro de una perspectiva valorativa, ha de dársele tanta importancia a los propósitos económicos relacionados con el aumento de lo que se produce, como a los propósitos sociales de distribución, sin descuidar los objetivos políticos de participación en las decisiones nacionales.

Los países pobres tienen la gran responsabilidad de integrar a su población a la comunidad nacional, especialmente a los que históricamente han sido excluidos. Una forma de integrarlos es garantizando salud, educación, empleo productivo y participación real en las decisiones políticas.

Este será un primer paso, muy importante para que las relaciones de interdependencia sean también justas y las naciones logren su inserción en la comunidad internacional en condiciones de igualdad.

Cambios de tal magnitud, implican cambios en las relaciones de poder, en la que no todos los grupos sociales estarán de acuerdo, por ello se necesita decisión política y voluntad nacional de salir del atraso y subdesarrollo.

El cambio social que implica un nuevo modelo de desarrollo basado en una economía de lo justo y austero implica también cambios estructurales que tendría gran significado sobre el control de los servicios institucionales.

Estamos ante un modelo excluyente que generaliza la pobreza y cualquier medida que se adopte si no va encaminada a impulsar cambios profundos en la estructura de producción, las formas de explotación de los recursos y los hábitos de consumo, solo servirá para aliviar la crisis que el mismo modelo genera. 


\section{REFERENCIAS}

Alburquerque, Eugenio. 2001. -Cambio Social ¿Cambio Moral? -Ediciones San Pablo, Madrid, España.

Boff, Leonardo. 2001. -Cuidar la tierra-Hacia una ética universal. -Ediciones DABAR S.A. de C.V. México, D.F.

Brisson, Margue.1999. -La globalización Capitalista...una exigencia de las ganancias. -Editorial Departamento Ecuménico de Investigaciones (DEI). -San José, Costa Rica.

Camacho, Ildefonso. 1991. -Doctrina Social de la Iglesia: Una aproximación histórica. -Ediciones San Pablo. -Sevilla, España.

Dierckxsens, Wim. 2004. -Otra economía es posible. -El futuro del mundo a corto, mediano y largo plazo. -Revista Pasos No 112.

Galindo, Ángel. 1996. -Moral Socioeconómica. -Biblioteca de Autores Cristianos (BAC). -Madrid.

Globalizar la Esperanza. -Fundación Amerindia. 1998. -Ediciones Dabar, México, D.F.

Goulet, Denis. 1995. -Ética del Desarrollo: guía teórica y práctica. Universidad de Notre Dame.

Hinkelammert, Franz. 2003. -El asalto al poder mundial y la violencia sagrada del imperio. - Colección Economía-Teología. -San José, Costa Rica.

Hinkelammert, Franz y Mora, Henry M. 2001. -Coordinación Social del trabajo, mercado y reproducción de la vida humana.-Colección Economía-Teología. -Editorial Departamento Ecuménico de Investigaciones (DEI), Costa Rica. 
Hughes, William. 1998. -Crecimiento y Desarrollo: desarrollo sostenible. -Memoria XI Congreso de Economistas de América Central y el Caribe. -Colegio Hondureño de Economistas, Tegucigalpa, Honduras C.A.

Keynes, John Maynard. 1981. -Teoría general de la ocupación, el interés y el dinero. -Fondo de Cultura Económica, México.

Kliksberg, Bernardo. 2002. -Hacia una economía con rostro humano. -Fondo de cultura económica. -Argentina.

Tablada, Carlos y Dierckxsens, Wim. 2003. -Guerra global, resistencia mundial y alternativas. Editorial de ciencias Sociales, La Habana.

Trputec, Zoran y Serrano, Augusto. 1997. -Hacia un concepto de desarrollo humano sostenible. Revista Paraninfo $\mathrm{N}^{0}$ 12, revista del Instituto de Ciencias del Hombre, Tegucigalpa, Honduras.

Verrier Castro, Roberto. -América Latina: el descalabro del modelo Neoliberal, y la crisis en el corazón de la economía norteamericana. -Fotocopiado. s.n.p.i. 